\title{
Perfil epidemiológico de fraturas em idosos no estado do Tocantins em uma década
} (2010 a 2020)

\author{
Epidemiological profile of fractures in the elderly in the state of Tocantins in a decade (2010 to
}

2020)

Perfil epidemiológico de fracturas en ancianos en el estado de Tocantins en una década (2010 a

2020)

Recebido: 23/09/2021 | Revisado: 30/09/2021 | Aceito: 07/10/2021 | Publicado: 10/10/2021

Kássio Maluar Gonçalves Luz

ORCID: https://orcid.org/0000-0002-4574-0251

Universidade Federal do Tocantins, Brasil E-mail: kassiomaluar@gmail.com

Fernanda Roques Felipe

ORCID: https://orcid.org/0000-0002-1661-6876 Universidade de Gurupi, Brasil

E-mail: nandaroquesf@gmail.com

Gisele de Jesus Batista

ORCID: https://orcid.org/0000-0001-9832-3307 Universidade de Gurupi, Brasil E-mail: gysabatysta@gmail.com

Carlos Alberto Rangearo Peres

ORCID: https://orcid.org/0000-0003-4064-5914

Universidade Federal do Tocantins, Brasil E-mail: carlos.peres@uft.edu.br

\begin{abstract}
Resumo
Introdução: Os idosos representam 14,6\% da população do estado Tocantins, chegando ao número de 228 mil pessoas. A ocorrência de fraturas neste público está relacionada à maior fragilidade óssea decorrente dos contextos fisiológicos degenerativos que aumentam o risco de quedas, vários fatores associados e predisponentes, sendo os mais frequentes os distúrbios neurológicos diversos, o uso de medicamentos que atuam sobre o psiquismo e que podem afetar o equilíbrio, o maior déficit muscular e a presença de artropatias e deficiências variadas decorrentes de diferentes doenças que podem estar presentes. Metodologia: Trata-se de uma pesquisa descritiva analítica, com abordagem quantitativa, em que se realizou busca de dados no Sistema de Informação do DATASUS no período de uma década de 2010 a 2020. Resultados: Foram encontrados um número de 7.382 fraturas em uma década no estado do Tocantins, eevidenciou um maior número de fraturas em idosos de 60 a 69 anos, com prevalência em mulheres e fraturas de outros membros. Conclusão: $\mathrm{O}$ maior número de fraturas foi encontrado no grupo de idosos mais jovens $(60$ a 69 anos), apresentando assim maior risco de trauma. A maior incidência de fratura em relação ao sexo foi o sexo feminino, e o maior número de fraturas encontradas em uma década foi de fraturas de outros ossos de membros.
\end{abstract}

Palavras-chave: Fraturas; Idosos; Vulnerabilidade de fraturas; Avaliação em saúde; Epidemiologia.

\begin{abstract}
Introduction: The elderly represents $14.6 \%$ of the population of the state of Tocantins, reaching a number of 228,000 people. The occurrence of fractures in this population is related to greater bone fragility resulting from degenerative physiological contexts that increase the risk of falls, several associated factors and predisposing factors, the most frequent being various neurological disorders, the use of drugs that act on the psyche and that can affect balance, greater muscle deficit and the presence of arthropathies and various deficiencies resulting from different diseases that may be present. Methodology: This is an analytical descriptive research, with a quantitative approach, in which data was searched in the DATASUS Information System over a decade from 2010 to 2020. Results: A number of 7,382 fractures were found in a decade in the state of Tocantins, which showed a greater number of fractures in the elderly aged 60 to 69 years, with a prevalence in women and fractures of other limbs. Conclusion: The highest number of fractures was found in the younger elderly group (60 to 69 years), thus presenting a higher risk of trauma. The highest incidence of fractures in relation to gender was female, and the largest number of fractures found in a decade were fractures of other limb bones.
\end{abstract}

Keywords: Fractures; Seniors; Fracture vulnerability; Health evaluation; Epidemiology. 


\begin{abstract}
Resumen
Introducción: Los ancianos representan el 14,6\% de la población del estado de Tocantins, llegando a 228.000 personas La ocurrencia de fracturas en esta población se relaciona con una mayor fragilidad ósea resultante de contextos fisiológicos degenerativos que incrementan el riesgo de caídas, varios asociados factores y factores predisponentes, siendo los más frecuentes diversos trastornos neurológicos, el uso de fármacos que actúan sobre la psique y que pueden afectar el equilibrio, mayor déficit muscular y la presencia de artropatías y diversas deficiencias derivadas de las distintas enfermedades que pueden estar presentes. Metodología:Se trata de una investigación descriptiva analítica, con enfoque cuantitativo, en la que se buscaron datos en el Sistema de Información DATASUS durante una década de 2010 a 2020. Resultados: Se encontraron 7.382 fracturas en una década en el estado de Tocantins, lo que evidenció mayor número de fracturas en ancianos de 60 a 69 años, con prevalencia en mujeres y fracturas de otros miembros Conclusión: El mayor número de fracturas se encontró en el grupo de ancianos más jóvenes (60 a 69 años), presentando así un mayor riesgo de traumatismo. La mayor incidencia de fracturas en relación con el sexo fue femenina, y el mayor número de fracturas encontradas en una década fueron fracturas de otros huesos de las extremidades.
\end{abstract}

Palabras clave: Fracturas; Personas mayores; Vulnerabilidad a la fractura; Evaluación de la salud; Epidemiología.

\title{
1. Introdução
}

O Instituto Brasileiro de Geografia e Estatística (IBGE) é um órgão responsável pelos levantamentos e gerenciamentos dos dados estatísticos brasileiros e registra que o Brasil acompanha a tendência mundial de envelhecimento da sua população. Com base nos dados de 2019, constatou-se que nos últimos quatro anos houve um aumento de 4,4 anos na expectativa de vida ao nascer da população masculina e de 0,7 para mulheres, chegando a 73,1 anos para homens e 80,1 anos para mulheres.

O envelhecimento de uma população, naturalmente, propicia aumento da prevalência de doenças neurovegetativas e psiquiátricas, além de enfermidades cardiovasculares, metabólicas e traumáticas, que segundo Chaimowwicz em 1997, acarreta crescimento dos pacientes que necessitam usar medicamentos de forma crônica, algumas vezes, sem acompanhamento médico rigoroso, para observar seus efeitos adversos e colaterais.

O IBGE em 2019 evidenciou um aumento nacional do número de idosos e com isso podemos trazer à tona uma discussão a respeito dos eventos incapacitantes nessa faixa etária, uma vez que as temidas quedas são bastante comuns com aumento na probabilidade de fraturas.

Paixão Júnior e Heckman (2002), relata que as fraturas por queda só ocorrerão se estiverem presentes, duas condições: perturbação do equilíbrio e falência por parte do sistema de controle postural, em compensar essa perturbação. Quanto mais frágil o paciente, mais suscetível a quedas e apresentando maior gravidade e maior probabilidade de fratura.

A pessoa idosa, naturalmente, passa a maior parte do tempo em sua residência. Esse ambiente pode parecer mais seguro ao seu morador, pela familiaridade com os supostos locais que aumentam os riscos de acidentes domésticos. Na segurança da própria residência, o residente considera que conhece o ambiente que vive, adquire uma autoconfiança e diminui a atenção para os locais de maior probabilidade para causar as quedas. Os hábitos de sua vida ativa passam a se constituir em perigos iminentes, tais como: alcançar objetos altos, trocar uma lâmpada, e até mesmo ir ao banheiro sozinho, podem resultar em uma queda (Mendes, et.al, 2005).

As atividades de vida diária (AVDs) das pessoas de maior idade exigem o continuísmo do bom desenvolvimento do equilíbrio e da marcha, que por sua vez, dependem de uma complexa interação entre as funções neurológicas, osteomusculares, cardiovasculares, além da capacidade de adaptar-se rapidamente às mudanças ambientais e posturais. Com o avançar da idade, o controle desse equilíbrio se altera, causa instabilidade na marcha, o que, associado à interação de vários fatores ambientais (superfícies irregulares, molhadas e desníveis no chão, escadas e tapetes) e do próprio indivíduo (patologias, alterações físicas e medicamentos) podem resultar em queda (Muniz, Arnaut, Yoshida, 2007).

Segundo o relatório global da Organização Mundial da Saúde (OMS) no ano de 2002, as quedas acontecem por perdas de equilíbrio postural, por problemas primários do sistema osteoarticular, neurológico ou mesmo de condições clínicas 
adversas que afetam secundariamente os mecanismos do equilíbrio e da estabilidade conforme fatores de riscos expostos. São elas: Físicos; Comportamentais; Psicológicos; Ambientais; Medicamentosos e doenças associadas e suas complicações (Freitas, et.al., 2015).

Em muitos casos os resultados dessas quedas são fraturas em diversos ossos. Fratura óssea é a perda da continuidade de um osso, e pode ser classificada em grande variedade de tipos. Elas podem ser múltiplas ou únicas, por encurtamento muscular violento ou por torção, completas ou incompletas, oblíquas, epifisárias, fechadas ou abertas, etc (ABCMED, 2013).

No idoso a maior ocorrência de fraturas, está relacionada à maior fragilidade óssea decorrente dos contextos fisiológicos degenerativos ou até mesmo por patologias como a osteoporose. As quedas, que ocorrem em maior número no ambiente doméstico, têm vários fatores associados e predisponentes, sendo os mais frequentes os distúrbios neurológicos, o uso de medicamentos que atuam sobre o psiquismo e que podem afetar o equilíbrio, o maior déficit muscular, e a presença de artropatias e as várias outras doenças relacionadas a este tema. Encontramos resultados semelhantes quanto ao principal local, ambiente doméstico, onde ocorreram as quedas e os medicamentos usados por este grupo (Suzuki, 2003).

O conjunto de todos esses riscos enfrentados diariamente pela pessoa idosa resultam em uma série de aspectos negativos em seu cotidiano, pois o idoso fica impedido de desfrutar de uma vida independente e ativa. Observa-se a falta de atenção dos idosos para tais limitações, o que os suscetibilizam as quedas. Apenas depois de vivenciá-las, recobram a consciência sobre a possibilidade de prevenção. Sendo assim, ações de domínio preventivo no processo interacional idoso, queda-fratura encontram-se num plano de invisibilidade mental, emergindo somente após o fato concretizado (Lopes, Dias, 2016).

Assim dentro das limitações e complicações encontrada na pessoa idosa, fica evidente a importância de conhecermos os principais tipos de fraturas nessa população, no estado do Tocantins, nos anos de 2010 a 2020, contextualizando seu perfil epidemiológico.

\section{Metodologia}

O presente estudo desenvolveu-se por meio de pesquisa com abordagem quantitativa, de caráter explicativo, onde foi realizada uma análise de dados documental em uma fonte limitada de coleta de informações. A população desse estudo foi todo paciente idoso que sofreu fratura, no estado do Tocantins, entre os anos de 2010 e 2020 e contabilizado no banco de dados do DATASUS.

A pesquisa quantitativa é aquela em que se coletam e analisam dados quantitativos sobre variáveis. Dessa forma, este tipo de pesquisa é capaz de identificar a natureza profunda das realidades, seu sistema de relações e sua estrutura dinâmica. Ela também pode determinar a força de associação ou correlação entre variáveis, a generalização e objetivação dos resultados através de uma mostra que faz inferência a uma população. Além do estudo da associação ou correlação, a pesquisa quantitativa também pode, ao seu tempo, fazer inferências causais que explicam por que as coisas acontecem ou não de uma forma determinada (Pita, Diaz, 2002).

Para obtenção desta análise foi empregado os seguintes questionamentos: número de pacientes idosos que sofreram fraturas, principais tipos de fraturas e sua divisão por sexo, divididos por décadas, de 60 a 69 anos; de 70 a 79 anos e 80 mais.

Para realização deste trabalho foram pesquisados dados referentes as fraturas em idosos no site nacional, o DATASUS, CID-10 no capítulo 19 Lista Morbidade - Lesões e outras consequências e causas externas --da categoria S027AT029, no estado do Tocantins do período de 2010 a 2020.

A existência de associações entre duas variáveis categóricas foi verificada utilizando-se o teste de Qui-Quadrado. Em se verificando diferenças na distribuição, foi utilizado o resíduo ajustado padronizado para identificar as diferenças locais com valores absolutos acima de 1,96 indicando evidências de associações (locais) entre as categorias relativas a essas caselas. As 
comparações de médias entre dois grupos foram realizadas utilizando-se o teste t de Student para amostras independentes. Para todos os testes estatísticos foram utilizados um nível de significância de $5 \%$.

\section{Resultados e Discussão}

A expectativa de vida do Brasileiro está aumentando. Os idosos representam 14,3\% da população nacional, chegando a 29,3 milhões de pessoas, em pesquisa divulgada pelo Instituto Brasileiro de Geografia e Estatística, no período de 2018 a 2019, a expectativa de vida da população brasileira, ao nascer, aumentou 3 meses, chegando em 2019 a 77,6 anos (IBGE, 2019).

Segundo dados da pesquisa nacional por amostra de domicílios do IBGE (2019) os idosos representam 14,6\% da população do estado Tocantins, chegando ao número de 228 mil pessoas.

O processo de envelhecimento traz consigo doenças crônico-degenerativas (Siqueira et al 2007). Neste contexto, concomitantemente, há um aumento dos fatores de risco e de mais comorbidades.

No Brasil, um terço dos indivíduos que chegam aos 70 anos apresenta doenças crônicas degenerativas não transmissíveis e pelo menos $20 \%$ desses idosos terá algum grau de incapacidade associada, implicando diminuição da capacidade física e restrições à autonomia e à independência (Ministerio da Saude, 2007).

$\mathrm{O}$ aumento da proporção de idosos na população brasileira traz à tona a discussão a respeito de eventos incapacitantes nessa faixa etária, dos quais se destaca a ocorrência de quedas, bastante comum e temida pela maioria das pessoas idosas por suas consequências, especialmente as fraturas (Paixao, Heckmann, 2002).

O idoso na maioria das vezes passa a maior parte do tempo em sua residência. Esse ambiente, por mais que pareça seguro pela familiaridade, pode tornar-se um ambiente de risco. Entre os acidentes domésticos, as quedas são as mais comuns em uma proporção de $70 \%$ e fatores externos em $30 \%$. Nesse ambiente, a pessoa idosa tem sua prontidão diminuída devido à autoconfiança para se deslocar, construída pelo conhecimento que possui sobre o ambiente em que vive. A atenção também fica reduzida porque as atividades que desempenha em seu lar são costumeiras, dessa forma, acidentes que poderiam facilmente ser evitados são causadores da redução da mobilidade ou da capacidade funcional. Muitas situações que eram corriqueiras, em sua juventude, passam a se constituir em perigos iminentes (Mendes, Gusmao, Faro, Leite, 2005).

De acordo com as Diretrizes da Sociedade Brasileira de Geriatria e Gerontologia, de 2008, aproximadamente 5\% dos episódios de queda desencadeiam fraturas, sendo as mais comuns são as vertebrais, no fêmur, úmero, rádio distal e costelas.

Rocha traz em seu trabalho no ano de 2005, que a fratura no idoso decorrente de queda é muito comum, dentre as fraturas ósseas a mais comum é a de fêmur. As fraturas aumentam a incidência de imobilização do idoso por um longo tempo ou também pode torná-lo um indivíduo acamado, acarretando modificações negativas na vida do idoso como a diminuição da funcionalidade, perda da autonomia e da independência. O tratamento desta fratura é em sua maioria cirúrgico. Fraturas de fêmur estão associadas à diminuição da qualidade de vida. Estudo realizado sobre evolução funcional nas fraturas de extremidade proximal do fêmur relata que os óbitos pós-fratura ocorrem nos primeiros meses e diminuem com o passar do tempo.

Uma fratura de fêmur pode ocorrer anatomicamente, na região proximal, diafisária e distal desse osso. Na maioria das vezes, cursam com complicações e consequências graves sobre a capacidade física, podendo comprometer a longevidade da pessoa acometida. Uma vez que o osso apresenta a capacidade de transmitir a carga durante a locomoção, com a fratura há perda da integridade estrutural óssea, o que prejudica a eficácia do movimento (Muniz, Arnaut, Yoshida, 2007).

Amarante et al., (2011) relata que a fratura de fêmur tem um potencial de interferência direta na qualidade de vida de um idoso. Na maioria dos casos os indivíduos desenvolvem uma dependência funcional, que acarreta limitações na realização 
de atividades cotidianas. A situação de dependência associado a várias comorbidades propiciam uma maior necessidade de intervenção cirúrgica, como tratamento.

Fraturas em pessoas idosas significam um grande desafio à saúde e qualidade de vida das mesmas. Fazendo-se uma projeção no perfil epidemiológico dos traumas com fratura, perspectivas futuras apontam que ao menos seis milhões de pessoas idosas irão sofrer fratura de fêmur até 2050. Tal projeção intensifica ainda mais a relevância deste estudo, permitindo assim observar esses números consideráveis de casos e evidenciar o péssimo cenário nacional (Soares ET.al., 2012).

Segundo Ishizuka, 2003, as quedas podem ter sérias consequências físicas e psicológicas, incluindo, hospitalizações, perturbação da marcha, medo de cair novamente, restrição da atividade, institucionalização e até mesmo a morte.

A presença de sintomas tais como: instabilidade postural, fraqueza dos membros inferiores, tonturas, audição deficiente, artrite, uso de psicotrópicos, sedativos e esteroides, podem provocar sérios riscos ao idosos e contribuir para a ocorrência de sérios agravos. A partir desse conhecimento, pode-se obter bons resultados preventivos ao promover ações que visem o bem-estar do idoso como um todo (Delduca et al., 2013).

Atualmente, as fraturas decorrentes de quedas são responsáveis por aproximadamente $70 \%$ das mortes acidentais em pessoas acima de 75 anos. Idosos apresentam dez vezes mais hospitalizações e oito vezes mais mortes consequentes de quedas (Konrad, Girardi, Helfert, 1999).

Os fatores de risco intrínsecos se relacionam ao próprio paciente e refletem incapacidade, pelo menos parcial, de manter ou de recuperar o equilíbrio quando houver um deslocamento acentuado do centro de gravidade localizado na região sacral. Tais fatores se referem à redução funcional em um ou mais sistemas sensórios-motores essenciais ao controle da postura, às doenças agudas ou crônicas, aos transtornos cognitivos e comportamentais, à fraqueza da musculatura dos membros inferiores e o uso de medicamentos (Guimaraes, Cunha, 2004).

Os fatores extrínsecos estão relacionados ao ambiente. Mais de $70 \%$ das quedas ocorrem em casa, e o risco se torna maior se o idoso vive só. Fatores ambientais podem ter um papel importante em até metade de todas as quedas. Portanto, torna se importante averiguar aspectos relacionados à: iluminação inadequada, superfícies escorregadias, tapetes soltos ou com dobras, degraus altos ou estreitos, obstáculos no caminho (móveis baixos, pequenos objetos, fios), ausência de corrimãos em corredores e banheiros, prateleiras excessivamente baixas ou elevadas, calçados inadequados e/ou patologias dos pés, maustratos e roupas excessivamente compridas (Hargreaves, 2006).

Conforme Soares e Custódio (2011), a população idosa tem estado cada vez mais presente nos hospitais, sendo essas hospitalizações normalmente mais prolongadas. Em meio a isso, há a necessidade de trabalhar com as especificidades dessa população, com apoio de uma equipe multidisciplinar, que estimule a promova um atendimento que supra as carências desse público, a fim de promover um cuidado mais sistematizado a esses indivíduos e diminuir seus períodos de internações ao mínimo de tempo possível.

Já a demora na realização do procedimento cirúrgico interfere na evolução da fratura, podendo modificar o tempo de permanência hospitalar, agregando elevados custos financeiros e aumentar a incidência de maneira significativa dos óbitos intra-hospitalares (Loures et al., 2015).

Mediante a esse contexto, esta pesquisa bibliográfica realiza o mapeamento quantitativo de casos de fraturas em idosos em uma década no estado do Tocantins, buscando evidenciar a incidência de fraturas no estado, como também realizar o perfil epidemiológicos desses pacientes.

Podemos acompanhar esses resultados obtidos através da pesquisa em DATASUS abaixo: 
Tabela 1. Fraturas em idosos no estado do Tocantins do período de 2010 a 2020.

\begin{tabular}{lccccccccccc}
\hline ANO & $\mathbf{2 0 1 0}$ & $\mathbf{2 0 1 1}$ & $\mathbf{2 0 1 2}$ & $\mathbf{2 0 1 3}$ & $\mathbf{2 0 1 4}$ & $\mathbf{2 0 1 5}$ & $\mathbf{2 0 1 6}$ & $\mathbf{2 0 1 7}$ & $\mathbf{2 0 1 8}$ & $\mathbf{2 0 1 9}$ & $\mathbf{2 0 2 0}$ \\
\hline TOTAL & 581 & 672 & 660 & 631 & 726 & 570 & 558 & 656 & 620 & 823 & 885 \\
\hline
\end{tabular}

Fonte: Ministério da Saúde - Sistema de Informações Hospitalares do SUS (SIH/SUS).

Como podemos ver, o número de fraturas na população idosa no estado do Tocantins é notório. Na Tabela 1, podemos notar uma diferença entre os números anuais de fraturas. Em sua maioria, notamos um aumento no número de casos de um ano para outro, mas também algumas discretas quedas como nos anos 2015/2016, onde houve uma queda de 2,1\% no número de fraturas.

O número acompanha oaumento da expectativa de vida da população idosa no estado Tocantinense, tendo um grande salto nos anos 2019 e 2020, pois se constata que nos últimos dois anos houve um aumento de 4,4 anos na expectativa de vida ao nascer da população masculina e de 0,7 para mulheres, chegando a 73,1 anos para homens e 80,1 anos para mulheres (IBGE, 2019).

Segundo estimativas, os idosos poderão atingir a cifra de 32 milhões de habitantes em 2025 esideses representando $15 \%$ da população do Brasil, diante disso fica evidente a necessidade de operacionalizar estratégias de prevenção de quedas nos idosos.

Do total de 228 mil idosos no estado do Tocantins no ano de 2020, um total de 0,38\% apresentou algum tipo de fratura, o que nos evidencia o maior número de fraturas em idosos neste período.

O ano de 2020 foi considerado um ano atípico pela presença da pandemia de COVID-19, os idosos permaneceram mais em casa, a mobilidade e espaço de locomoção ficaram diminuídos, o que pode ser percebido pelo aumento de 7,5\% no número de casos de fraturas, desse grupo, comparado ao ano anterior. (IBGE, 2019)

Segundo Arliani (2011), as quedas em idosos, podem estar relacionadas à presença de comorbidades clínicas, a maior tempo de internação e ainda a complicações advindas de procedimentos cirúrgicos, como a anestesia geral, que provocam sonolência, alteram o equilíbrio, a tonicidade muscular e provoca hipotensão. Como exemplo de medicamentos utilizados na anestesia geral, citamos o uso de anti-hipertensivos que podem provocar hipotensão postural ou mesmo tonturas; e o uso de diuréticos, fazendo com que o paciente levante à noite para urinar e facilite quedas e consequentes fraturas.

Tabela 2. Fraturas em idosos, divisão por sexo, no estado do Tocantins do período de 2010 a 2020.

\begin{tabular}{|c|c|c|c|c|c|c|c|c|c|c|c|c|}
\hline SEXO & $\underline{2010}$ & $\underline{2011}$ & 2012 & 2013 & 2014 & 2015 & 2016 & 2017 & 2018 & 2019 & 2020 & Total \\
\hline Masc. & 272 & 326 & 302 & 288 & 347 & 283 & 274 & 317 & 279 & 403 & 452 & 3543 \\
\hline Fem. & 309 & 346 & 358 & 343 & 379 & 287 & 284 & 339 & 341 & 420 & 433 & 3839 \\
\hline
\end{tabular}

Fonte: Ministério da Saúde - Sistema de Informações Hospitalares do SUS (SIH/SUS).

Nesta tabela discutiremos o número de casos de fraturas segregados por sexo. Ao longo da década, fica notável a dominância feminina no número de casos de fraturas. Somente no ano de 2020 tivemos uma dominância masculina com uma porcentagem de $51,07 \%$ de total de casos de fraturas.

As mulheres possuem 52\% de casos de fraturas em uma década (2010-2020) no estado do Tocantins.

Estudo de Perracini e Ramos (2002), obteve resultados semelhantes quanto a prevalência do sexo feminino, possuindo maior incidência de fraturas, se associada a outras variáveis como: história prévia de fratura e ter dificuldade na execução das atividades físicas e possuir visão ruim ou péssima. O mesmo estudo traz ainda, as possíveis causas para maior ocorrência de 
quedas em mulheres, são a maior fragilidade destas em relação aos homens, assim como maior prevalência de doenças crônicas. Suspeita se, ainda, que o fato pode estar relacionado a uma maior exposição a atividades domésticas e a um comportamento de maior risco.

Essa evidente suscetibilidade do gênero feminino chama a atenção para o problema da osteoporose, que é uma doença sistêmico-progressiva caracterizada pela diminuição da massa óssea e deterioração da microarquitetura, levando à fragilidade do osso e aumentando o risco de fraturas (Gali, 2001).

Tabela 3. Fraturas em idosos, faixa etária, no estado do Tocantins do período de 2010 a 2020.

\begin{tabular}{|c|c|c|c|c|c|c|c|c|c|c|c|}
\hline $\begin{array}{c}\text { FAIXA } \\
\text { ETÁRIA }\end{array}$ & 2010 & 2011 & 2012 & 2013 & 2014 & 2015 & 2016 & 2017 & 2018 & 2019 & 2020 \\
\hline \multicolumn{12}{|l|}{60 a 69} \\
\hline Anos & 258 & 276 & 280 & 262 & 325 & 267 & 250 & 301 & 302 & 373 & 404 \\
\hline \multicolumn{12}{|l|}{70 a 79} \\
\hline Anos & 183 & 201 & 206 & 198 & 222 & 165 & 156 & 209 & 188 & 218 & 258 \\
\hline $\begin{array}{l}80 \text { anos e } \\
\text { mais }\end{array}$ & 140 & 195 & 174 & 171 & 179 & 138 & 152 & 146 & 130 & 232 & 223 \\
\hline TOTAL & 581 & 672 & 660 & 631 & 726 & 570 & 558 & 656 & 620 & 823 & 885 \\
\hline
\end{tabular}

Fonte: Ministério da Saúde - Sistema de Informações Hospitalares do SUS (SIH/SUS).

$\mathrm{Na}$ Tabela 3, teremos a divisão do número de casos de fraturas distribuídos em 3 faixas etárias: a primeira no idoso com idade entre 60 e 69 anos, que apresenta um número evidentemente maior de fraturas, com 45,64\% dos casos totais de fratura; a segunda de idosos de 70 a 79 anos, apresentam 29,15\% dos casos de fratura, um número bem menor que a primeira faixa etária; e a terceira e mais velha em anos (80 anos e mais) foi representada por 11,98 \% dos casos de fraturas nos idosos.

Os dados encontrados no Tocantins contrariam os resultados de outros estudos brasileiros, pois a primeira faixa etária, idosos mais jovens, apresentou um maior número de fraturas. Segundo estudo realizado por Gawryszewski (2010), em relação à faixa etária com maior ocorrência de quedas, encontrou dados que verificaram maior incidência de quedas entre os idosos mais velhos, entre idades de 80 anos ou mais, em relação aqueles com idade entre 60 e 79 anos. Os resultados confirmam que idosos acima de 80 anos apresentam mais riscos relativos de quedas do que idosos de faixa etária inferior.

Uma pesquisa realizada por Pereira, no ano de 2001 trouxe que os idosos caem mais em seu próprio lar do que na rua ou em outros ambientes, em uma proporção de $70 \%$ das quedas ocorrem no interior da residência, sendo que as pessoas que vivem sozinhas apresentam maior risco (Pereira, 2001).

Estudo de Perracini e Ramos (2002), contesta o achados deste trabalho que verificou que idosos de 60 a 69 anos apresentaram mais fraturas em uma década,eles afirmaram em suas pesquisas que idosos com idade superior a 80 anos estão 14 vezes mais propensos a cair (expondo-se a riscos de lesões e fraturas decorrentes das quedas) do que idosos com idade inferior. Segundo os pesquisadores, esse fato ocorre provavelmente porque os idosos com 85 anos ou mais diminuem suas atividades naturalmente (Freitas, Scheicher, 2008). 
Tabela 4. Locais de Fraturas em idosos, CID 10, no estado do Tocantins do período de 2010 a 2020.

\begin{tabular}{|c|c|c|c|c|c|c|c|c|c|c|c|c|}
\hline ANO & 2010 & 2011 & 2012 & 2013 & 2014 & 2015 & 2016 & 2017 & 2018 & 2019 & 2020 & TOTAL \\
\hline \multicolumn{13}{|c|}{$\begin{array}{c}\text { Fratura do crânio } \\
\text { e dos ossos da } \\
\text { face }\end{array}$} \\
\hline \multicolumn{13}{|c|}{$\begin{array}{l}\text { Fratura do } \\
\text { pescoço } \\
\text { tórax ou pelve }\end{array}$} \\
\hline & 17 & 17 & 28 & 33 & 30 & 18 & 23 & 23 & 21 & 15 & 20 & 245 \\
\hline \multicolumn{12}{|l|}{ Fratura do } & 2276 \\
\hline \multicolumn{13}{|c|}{$\begin{array}{l}\text { Fratura de } \\
\text { outros ossos } \\
\text { dos membros }\end{array}$} \\
\hline & 320 & 348 & 303 & 310 & 333 & 274 & 252 & 309 & 309 & 374 & 411 & 3543 \\
\hline \multicolumn{13}{|c|}{$\begin{array}{l}\text { Fraturas } \\
\text { envolvendo } \\
\text { múltiplas regiões } \\
\text { do }\end{array}$} \\
\hline corpo & 55 & 47 & 68 & 68 & 95 & 90 & 95 & 124 & 101 & 199 & 174 & 1116 \\
\hline \multicolumn{13}{|l|}{ TOTAL } \\
\hline & 581 & 672 & 660 & 631 & 726 & 570 & 558 & 656 & 620 & 823 & 885 & 7382 \\
\hline
\end{tabular}

Fonte: Ministério da Saúde - Sistema de Informações Hospitalares do SUS (SIH/SUS).

Na tabela 4 podemos observar a distribuição dos locais de fraturas pelo CID 10.

A maior incidência nos casos de fraturas em idosos foi as Fratura de outros ossos dos membros com dominância em todos os anos da década, apresentando um total de 47,99\% das fraturas registradas em uma década. Seguida pela fratura de fêmur com 30,83\% dos casos de fraturas. Se somarmos as duas, fratura de outros ossos dos membros e fratura de fêmur chegou a $78,82 \%$ dos casos de fratura. Com $15,11 \%$ das fraturas em múltiplas regiões do corpo, seguidas pela fratura de pescoço, tórax ou pelve com 3,31\% e fratura de crânio e ossos face com $2,7 \%$ dos casos registrados.

\section{Conclusão}

O aumento da expectativa de vida traz consigo uma maior probabilidade da presença de comorbidades e complicações da saúde como as fraturas, que complicam a continuidade da vida e diminuem a qualidade de vida desse grupo.

Ao concluirmos esta pesquisa epidemiológica, onde avaliamos o número de fraturas em idosos, divididas por sexo e faixa etária, trazendo ainda os tipos de fraturas encontrados, concluímos que há uma maior evidência do número de fraturas em idosos mais jovens (60 a 69 anos).

Quando dividimos essa população pelo sexo, encontramos uma maior incidência de fratura entre mulheres, que como referenciado nesta pesquisa, possuem maior dever em atividades domésticas e maiores fragilidades metabólicas.

Ao contabilizarmos o quantitativo de fraturas em idosos divididos em locais de fraturas, concluímos que os maiores números de fraturas encontradas durante a década nos anos de 2010 a 2020 foram de fraturas de outros ossos de membros, seguidos por fraturas de fêmur.

E se analisarmos que o fêmur é um osso de membro, podemos somar suas fraturas as de ossos de membro, chegando a apresentar um total de $78,82 \%$ das fraturas registradas em uma década. 
Em síntese este trabalho visou realizar um estudo que evidenciasse o número de fratura na população do estado do Tocantins na última década, avaliando os anos de 2010 a 2020, pois sabemos a grande vulnerabilidade e riscos de fraturas que a população idosa sofre ao longo do processo de envelhecer.

É de extrema importância trabalhos científicos que visem a melhoria da qualidade de vida dos idosos, identificando a incidência de fraturas, os seus tipos de fraturas, o sexo e idade que mais sofrem com esses traumas, incluindo dados que explanem a realidade das patologias encontradas por esse frágil grupo, pois somente assim, podemos elaborar intervenções efetivas para mudança desse cenário.

\section{Referências}

ABCMED. (2013). Fratura óssea: definição, causas, sinais e sintomas, tipos de fraturas, diagnóstico, tratamento e evolução. https://www. abc.med.br/p/ortopedia-e-saude/370949/fratura-ossea-definicao-causassinais-e-sintomas-tipos-de-fraturas-diagnostico-tratamento-e-evolucao.htm.> .

Arliani, G. G. Astur, D. C., Linhares, G. K. Balbachevsky, D., Fernandes, H. J. A. Reis, F. B. (2011). Correlação entre o tempo cirúrgico e mortalidade em pacientes idosos com fratura de extremidade proximal do fêmur. Rev Bras Ortop.

Amarante, C. F. S. et al. (2011). Fraturas do fêmur proximal em idosos. Revista de Medicina de Minas Gerais, Belo Horizonte M.G. 21(2), 21-24

Brasil. Ministério da Saúde (MS). Secretaria de Atenção Básica. Departamento de Atenção Básica. Envelhecimento e saúde da pessoa idosa Brasília: MS, 2007. (Série A. Normas e Manuais Técnicos).

Chaimowicz F. (1997) A saúde dos idosos brasileiros às vésperas do século XXI: problemas, projeções e alternativas. Rev Saúde Pública. 31:184-200.

Cruz, D. T., et al. Prevalência de quedas e fatores associados em idosos. Rev Saude Pública.

Delduca, G. F., et al. Quedas e fraturas entre residentes de instituições de longa permanência para idosos. Revista Brasileira de Epidemiologia, 16(1), 68-76.

Freitas, M. A. V., \& Scheicher, M. E. (2008) Preocupação de idosos em relação a quedas. Rev Bras Geriatr Gerontol. 11(1):57-64

Gali, J. C. (2001) Osteoporose. Acta Ortop Bras. 9: 53-62.

Gawryszewski, V. P. (2010) A importância das quedas no mesmo nível entre idosos no Estado de São Paulo. Rev Assoc Med Bras. 56(2):162-7.

Guimarães, R. M., \& Cunha, U. G. V. (2004) Sinais e sintomas em geriatria. (2a ed.), Atheneu.

Gomes, E. C., Marques, A. P., Leal, M. C., \& Barrs, B. P. (2014) Fatores associados ao risco de quedas em idosos institucionalizados: uma revisão integrativa. Ciênc Saúde Colet.

Hargreaves, L. H. H. (2006) Geriatria. Brasilia

IBGE. Instituto Brasileiro de Geografia e Estatística IBGE. (2019) Censo demográfico: resultados preliminares. IBGE.

Ishizuka, M. A. (2003) Avaliação e comparação dos fatores intrínsecos dos riscos de quedas em idosos com diferentes estados funcionais [dissertação]. Campinas: Faculdade de Educação, Universidade Estadual de Campinas, 10.

Konrad, H. R., Girardi, M., \& Helfert, R. (1999) Balance and aging. Laryngoscope. 109(9): 454-60.

Loganathan, A., NG, C. J., Low W. Y. (2016) Views and experiences of Malaysian older persons about falls and their prevention: a qualitative study. BMC Geriatr.

Lopes, R. A., \& Dias, R. C. O (2010) impacto das quedas na qualidade de vida dos idosos. Rev ConScientiae Saúde. 9(3):504-9. http://www.redalyc.org/pdf/929/92915180022.pdf

Loures, F. B. et al $\left(2015^{\mathrm{a}}\right)$. Análise econômica do tratamento cirúrgico de fratura do quadril em idosos. Revista de Saúde Pública, 49 , 12.

Martins, G. A. \& Pinto, R. L. (2001) Manual para elaboração de trabalhos acadêmicos. Atlas.

Mendes, M. R. S. S., et al. (2005) A situação social do idoso no Brasil: uma breve consideração. Acta Paul Enfermagem. 18(4):422-6.

Muniz, C. F., Arnaut, A. C., \& Yoshida, M. (2007) Caracterização dos idosos com fratura de fêmur proximal em hospital escola público. Rev Espaço Saúde. $8(2): 33-8$.

Paixão, J., \& Heckmann, M. (2002) Distúrbios da postura, marcha e quedas. In: Freitas EV, Py L, organizadores. Tratado de Geriatria e Gerontologia. Guanabara Koogan, 624-34.

Pereira, S. E. M., et al. (2001) Quedas em idosos. Rio de Janeiro: Sociedade Brasileira de Geriatria e Gerontologia, http://www.sbgg.org.br/profissionais/index.php?diretrizes

Perracini, M. R., \& Ramos, L. R. (2002) Fatores associados a quedas em uma coorte de idosos residents na comunidade. Revista de Saúde Pública. 36(6):70916. 
Research, Society and Development, v. 10, n. 13, e206101320986, 2021

(CC BY 4.0) | ISSN 2525-3409 | DOI: http://dx.doi.org/10.33448/rsd-v10i13.20986

Pita Fernández, S., \& Pértegas Díaz S. Investigación cuantitativa y cualitativa. Cad Aten Primaria, 9, 76-8.

Rarnett, A., et al. (2003) Community-based group exercise improves balance and reduces falls in at-risk older people: a randomized controlled trial. Age Ageing. 32(4):407-14

Rocha, M. A. A., et al. (2009) Evolução funcional nas fraturas da extremidade proximal do fêmur. Acta ortopédica brasileira, 17(1), 17-21.

Siqueira, F. V., et al. (2007) Prevalência de quedas em idosos e fatores associados. Rev Saude Publica. 41(5):749-756.

Secretaria de Estado de São Paulo (SP). Relatório global da OMS sobre prevenção de quedas na velhice. Centro de Produção e Divulgação Científica.

Soares, D. S., et al. (2015) Análise dos fatores associados a quedas com fraturas de fêmur: um estudo caso controle. Revista Brasileira de Geriatria. Gerontol, 18(2) 239-248. http://dx.doi.org/10. 1590/1809-9823.2015.14022 >.

Soares, D. S. M. et al. (2015) Análise dos fatores associados a quedas com fratura de fêmur em idosos: um estudo caso-controle, Rev. Bras. Geriatr. Gerontol.,

SBGG. Sociedade Brasileira de Geriatria e Gerontologia.Queda em Idosos: prevenção.Projeto Diretrizes.2008

Suzuki, I. (2003). Alterações ortopédicas em geriatria. In: Herbert S, Xavier R. Ortopedia e Traumatologia: princípios e prática. (3a ed.), Artmed. 766-70.

WTO/WHO (World Trade Organization/ 0World Health Organization). 2002. World Trade Organization agreements Public Health: a joint study by the WHO and the WTO Secretariat. 